\title{
El adulto mayor y la universalidad de la salud: Análisis de desigualdad basado en una comparación entre los diferentes grupos de edad $^{*}$
}

\author{
KARLA HERNÁNDEZ-VILLAFUERTE \\ Office of Health Economics, Southside 7th Floor, 105 Victoria Street, London SWIE 6QT, United \\ Kingdom. E-mail: KHernandez-Villafuerte@ohe.org
}

\begin{abstract}
RESUMEN
Costa Rica cuenta con uno de los mejores sistemas de salud de Latinoamérica. Sin embargo, debido a la relación positiva entre el nivel socioeconómico y la salud (comprobada en estudios anteriores), la interacción entre el reciente incremento en la desigualdad socioeconómica y el envejecimiento poblacional hace temer la aparición de desigualdades en salud. El presente análisis tiene como objetivo examinar las desigualdades en salud en Costa Rica, comparando las diferencias existentes en el grupo de adultos mayores de 60 años y los demás grupos de edad. La desigualdad se mide mediante el Índice de Concentración de Erreygers. Para explicar los determinantes de la desigualdad en salud se descompone el índice en los posibles factores que lo explican. Los resultados indican que existe desigualdad en salud que afecta negativamente a los más pobres. Las desigualdades son no significativas en los adultos mayores de 70 años y significativas entre los 50 y 69 años.
\end{abstract}

Palabras clave: Grupos de edad, desigualdad en salud, índice de concentración, Costa Rica.

\section{The Elderly and the Universality of Health: An Inequality Analysis Based on a Comparison between Age Groups}

\begin{abstract}
Costa Rica accounts for one of the best healthcare systems in Latin-American. However, the combination between the increase in socioeconomic inequalities and the ageing of the population raises some concerns about the possibility of an increase in healthcare inequalities. This is a consequence of the positive relationship between socioeconomic conditions and health status which has been demonstrated in previous studies. Therefore, the aim here is to examine the inequalities in health presented in Costa Rica, and to compare the differences between elderly (aged 60 or over) and other age groups. Health Inequalities are measured through the Erreygers Concentration Index. This index is broken down into individual factors that could make a significant contribution to the creation of inequalities in health. Significant socioeconomic inequalities in health have been found, with the proportion of healthy people higher among the rich than among the poor, with differences being particularly significant between 50 and 69 year olds.

Keywords: Age Groups, Inequalities, Concentration Index, Costa Rica.

Clasificación JEL: I14, I13, I15

\footnotetext{
* Los datos de la Encuesta Nacional de Salud de Costa Rica fueron suministrados gratuitamente por el Centro Centroamericano de Población de la Universidad de Costa Rica, quienes también proporcionaron soporte financiero.

Agradecimientos: Al profesor Juan Rafael Vargas de la Universidad de Costa Rica quien ha aportado tiempo e ideas en la elaboración de este trabajo. Agradecer el apoyo económico y técnico brindado por la "Alliance for Health Policy and Systems Research" de la OMS.
}

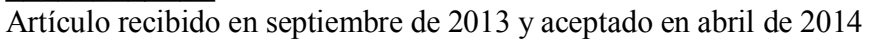

Artículo disponible en versión electrónica en la página www.revista-eea.net, ref. ə-32302 


\section{INTRODUCCIÓN}

Las desigualdades en salud son una realidad tanto en países en desarrollo como desarrollados (Marmot, 2005) (Murray et al., 2006). El vínculo con el nivel de ingreso es innegable, desigualdades en salud, donde los individuos pertenecientes a los extractos más bajos de ingreso presentan peores condiciones de salud que los de más altos de ingresos, han sido observadas independientemente del sistema de seguridad social o de las características culturales y/o económicas (Mackenbach et al., 2008; Wagstaff et al., 1991). Costa Rica, pese a contar con uno de los mejores sistemas de salud en Latinoamérica (OMS, 2000), no es la excepción (Cardona et al., 2013). Morera (Morera, 2009), basándose en los datos de la Encuesta Nacional en Salud, muestra como el porcentaje de costarricenses que reportan estados de salud "no buenos" aumenta en un $15 \%$ al pasar de los hogares más ricos a los más pobres. Esta relación entre el nivel de salud y el nivel de ingreso es un tema trascendental para Costa Rica ya que las desigualdades socioeconómicas han aumentado en los últimos años de manera considerable (Estado de la Nación, 2012). Esto se comprueba al observar la evolución del índice de Gini, el cual paso de ser 0,37 en 1990 hasta alcanzar un máximo histórico de 0,51 en el 2011 (Estado de la Nación, 2012). Como lo sugiere la evidencia empírica (Hernández-Quevedo y Masseria, 2013), esta tendencia puede llegar a ensanchar la brecha en las condiciones de salud entre los costarricenses ricos y pobres. A pesar de esto, solo se conoce un estudio que ha medido el nivel de desigualdades socioeconómicas en salud en Costa Rica pero sin analizar sus causas (Cardona et al., 2013). Esto dificulta la respuesta de los hacedores de las políticas de salud ante el incremento en la desigualdad. Con el objetivo de llenar este vacío de conocimiento, el presente análisis busca investigar la relación entre el nivel de ingreso y el estado de salud para el caso costarricense. Antes de iniciar el análisis, se introduce en los próximos párrafos las principales características del sistema de salud costarricense que se consideran factores claves para abordar el estudio de las desigualdades socioeconómicas en salud.

Una característica significativa del sistema de salud costarricense es que basa en los principios de universalidad y solidaridad. La Ley General de Salud estipula que una función esencial del Estado es velar por la salud de los costarricenses garantizando el derecho de las prestaciones de salud a todos los habitantes del país (Asamblea Legislativa de Costa Rica, 1973). Como resultado un $85.5 \%$ de la población se encuentra asegurada (INEC, 2011) y el $14.5 \%$ restante puede hacer uso de los servicios de salud públicos a través de la atención de emergencias (Saénz et al., 2011). La reforma del sector salud iniciada en 1995 ha sido un factor clave para alcanzar estos elevados niveles de cobertura, facilitando además el acceso a los servicios de salud públicos (Rosero, 2004). La pieza central de esta reforma son los denominados equipos 
básicos de atención integral de la salud (EBAIS). Estos centros prestan servicios en el primer nivel de atención y cada uno es responsable por la salud de alrededor de 4000 personas (Rosero, 2004). Actualmente existen 1014 EBAIS dispersos por todo el país los cuales además realizan labores de promoción de hábitos de vida saludable (CCSS, 2013).

A pesar de las mejoras en el acceso y la cobertura de los servicios públicos de salud, no está claro si la reforma del sector ha disminuido la desigualdad en salud. Esto debido a que acceso y cobertura son condiciones necesarias para disminuir las desigualdades pero no son suficientes. Esto se ve corroborado con los resultados del estudio de Mackenbach et al. (2008), los cuales indican que así como la salud es multifactorial, lo es también la desigualdad, por lo que se ve afectada por los hábitos y características relacionadas a los niveles socioeconómicos. Consecuentemente, el nivel de desigualdad en Costa Rica podría estarse viendo afectado por la incidencia de factores que se consideran de riesgo para la salud, como por ejemplo fumar, ingerir bebidas alcohólicas y tener sobrepeso, los cuales son un problema de salud pública en Costa Rica más frecuente entre personas de menores niveles de ingreso. Por otra parte, la elevada cobertura del servicio público de salud ha traído a su vez largas listas de espera en el segundo y tercer nivel de atención. Esto ha causado el descontento de los usuarios ante los servicios de la Caja Costarricense del Seguro Social (CCSS), institución pública responsable de brindar los servicios de salud, lo que ha tenido como consecuencia un incremento en el uso de los servicios privados (Herrero y Durán, 2001). Si bien no existe aún gasto catastrófico alto en Costa Rica (Knaul et al., 2011), este deterioro en el sistema público ha llevado a que un $30 \%$ del gasto en salud sea provisto por el sector privado y financiado principalmente por recursos de bolsillo (Herrero y Durán, 2001). Los costarricenses de mayores ingresos son los que con más frecuencia toman la decisión de evitar las listas de espera y acudir a la consulta privada, lo cual genera una brecha en tiempo de espera entre los ricos y pobres (Herrero y Durán, 2001).

Otra característica importante en el sistema de salud costarricense es la distribución por edad de los usuarios. En Costa Rica el mejoramiento en la disponibilidad de los servicios de salud y las condiciones de vida ha provocado un cambio en la estructura demográfica. La esperanza de vida al nacer es comparable a la de los países desarrollados, siendo igual a 76.5 años para los hombres y 81.0 para las mujeres. A raíz de esto, los adultos mayores de 60 años son quienes utilizan más los servicios de salud tanto públicos como privados (Fernández y Robles, 2008a). Tendencia que se prevé se acentuará en los próximos años, ya que actualmente $9.7 \%$ de la población tiene más de 65 años (Estado de la Nación, 2013) y se estima que para el 2060 este valor ascenderá a casi un 25\% (CCP y INEC, 2008). El mayor uso de los servicios de salud por parte de los adultos mayores es un tema trascendental cuando hablamos de desigualdades en 
salud. La literatura sugiere que entre el grupo de los adultos mayores ${ }^{1}$ las desigualdades en salud relacionadas a las condiciones socioeconómicas son menores en comparación con los otros grupos de edad (Deaton y Paxson, 1998; Grundy y Sloggett, 2003; Huisman et al., 2003; Van Ourti, 2003). Deaton y Paxson (1998) explican esta discrepancia como el resultado de las diferencias entre los pensionados y las personas en la fuerza laboral. Ellos argumenta que la oferta de trabajo y la capacidad para obtener ingresos son afectadas de manera negativa y acumulativa por los problemas de salud, por lo cual conforme se incrementa la edad y surgen mayores problemas de salud la correlación negativa entre ingreso y salud aumenta; sin embargo, en vista de que los problemas de salud no afectan el pago de las pensiones o la seguridad social, esta correlación se debilita en los grupos de mayor edad (Deaton y Paxson, 1998). Por su parte Huisman et al., 2003 proponen que el ingreso monetario no es una medida adecuada de la capacidad financiera de los adultos mayores. Muchas personas al llegar a edades por encima de los 60 años han acumulado una serie de posesiones durante la vida que no siempre se ven reflejadas en el ingreso, como por ejemplo la tenencia de una casa propia. No está claro a-priori que en Costa Rica se cumpla que la desigualdad sea menor para los grupos de mayor edad. Los hogares costarricenses con personas adultas mayores registran un índice de pobreza superior, sin embargo, para aquellos hogares con adultos mayores que poseen ingresos provenientes de pensiones o de riqueza propia el riesgo de pobreza es más bajo (Fernández y Robles, 2008b). Por tanto, el análisis de las desigualdades en salud en Costa Rica debe tomar en consideración las diferencias por grupo de edad. Cualquier intento de política que busque la igualdad pero que no tome en cuenta las variaciones en la desigualdad según grupo edad podría llevar a resultados no deseados.

El objetivo central del presente documento es analizar la desigualdades socioeconómicas en salud existentes en el grupo de adultos mayores de 60 años en Costa Rica, además de hacer una comparación entre estas y las desigualdades de los otros grupos de edad. Adicionalmente, se examinan los posibles factores que contribuyen a la desigualdad y se identifica la importancia de los mismos según el grupo de edad. Con este fin se estima el Índice de Concentración en Salud (O'Donnell et al., 2008; Kakwani et al., 1997) ajustado por la modificación hecha por Erreygers (2009). Además, se aplica la metodología propuesta por Rao (1969) y aplicada a la descomposición del índice por van Doorslaer et al. (2004) y Wagstaff et al. (2003) la cual permite identificar los factores que contribuyen a la desigualdad.

Se utiliza como medida de desigualdad socioeconómica el nivel de ingreso per-capita familiar. La desigualdad en la distribución del ingreso en Costa Rica

\footnotetext{
${ }^{1}$ No existe un consenso en la literatura sobre a partir de cual edad una persona debe ser considerada adulto mayor, lo común es utilizar 60 o 65 años.
} 
continúa creciendo con una clara tendencia a la concentración en los grupos más adinerados. Por este motivo, enfrentar el impacto del incremento en la brecha de ingreso entre ricos y pobres ocupa un lugar prioritario en la agenda política. Por tanto, usar el ingreso per-capita familiar como medida del estatus socioeconómico es consistente con los objetivos de política planteados por el gobierno. Por otra parte, los resultados del estudio dirigido por Wagstaff y Watanabe (2003), sugieren que la medida del estatus socioeconomico utilizada tiene poco impacto en la estimación de las desigualdades socieconomicas en salud.

\section{METODOLOGÍA}

\subsection{Estimación de las Desigualdades en Salud}

Con el objetivo de medir la desigualdad se analiza el índice de concentración (IC) (O'Donnell et al., 2008). Este índice cumple con los requisitos mínimos necesarios para medir adecuadamente la desigualdad en salud: refleja no solo la situación del primer y último grupo de ingreso sino de toda la población, además refleja las dimensiones socioeconómicas de las desigualdades y es sensible a cambios en la distribución de la población entre los diferentes grupos socioeconómicos (Wagstaff et al., 1991).

Figura 1

Curva de Concentración (CC)

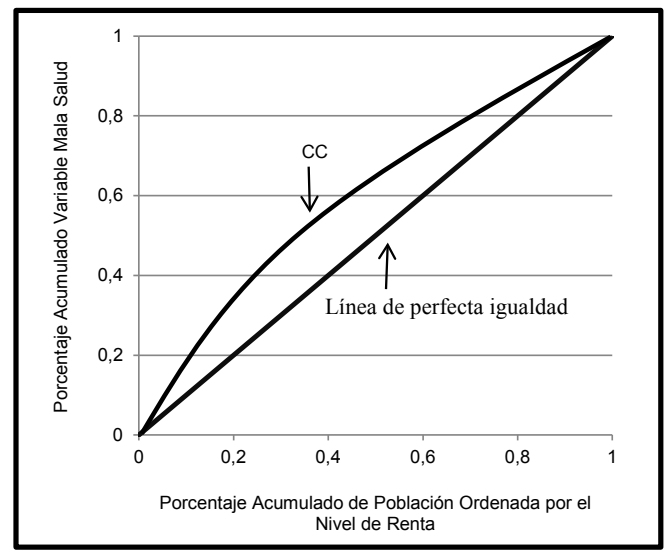

Fuente: Elaboración propia.

El IC se deriva de un concepto más intuitivo y que se analiza gráficamente, la curva de concentración (CC). La CC presenta la proporción acumulada de observaciones dentro de cada grupo de ingreso ordenado por nivel de renta contra el porcentaje acumulado de salud correspondiente (Figure 1). El IC es definido como dos veces el área entre la $C C$ y la línea de perfecta igualdad. Por tanto en aquellos casos en los cuales no existe desigualdad en la distribución de 
la salud por nivel de ingreso el índice de concentración es cero. El IC se distribuye entre $[-1,1]$, índices de concentración igual a 1 indican que la salud está totalmente concentrada en los más ricos de la sociedad. Si la medida de salud está desproporcionadamente concentrada en los niveles más bajos de ingresos, la curva se encontrará por encima de la línea de perfecta igualdad y el índice presentará valores negativos.

Con el fin de utilizar al máximo la información de la asociación entre ingreso y salud, el $I C$ es generalmente calculado usando los datos asociados a cada individuo y no con datos agrupados. Sin embargo, esto supone que la información con la que se cuenta permite obtener un único ordenamiento de los individuos según su nivel de ingreso. Esto no es posible cuando varios individuos reportan el mismo nivel de ingreso (Clarke y Van Qurti, 2009). En este caso es necesario agrupar las observaciones según nivel de ingreso. El IC para datos agrupados se define matemáticamente como:

$$
I C=\frac{2}{\mu} \sum_{t=1}^{T} f_{t} \mu_{t} R_{t}-1 \quad(t=1, \ldots, T)
$$

Una vez ordenados los grupos de menor $(t=1)$ a mayor $(t=\mathrm{T})$ de acuerdo con su ingreso $f_{t}$ es el porcentaje de individuos en el grupo $t$ y $\mu_{\mathrm{t}}$ es el promedio de la variable salud dentro de ese grupo. $\mu$ es el promedio de la variable salud para toda la muestra. $R_{t}$ es la posición relativa del grupo $t \mathrm{y}$ se define como $\left(\sum_{l=1}^{t-1} f_{l}+\frac{1}{2} f_{t}\right)$ (Jenkins, 1988; Kakwani, 1980; Kakwani et al., 1997).

Como se mencionó anteriormente, parte del objetivo del presente documento es analizar la desigualdad por grupo de edad y ahondar en la situación comparativa de los adultos mayores. Para cumplir con este objetivo es necesario reconocer si las desigualdades en salud son significativamente diferentes de cero y si las diferencias entre los grupos de edad son significativas. Con este fin se evalúa el intervalo de confianza al $95 \%$ para el IC. Este se basa en la fórmula propuesta por Kakwani et al. (1997) para el cálculo de la varianza:

$$
\operatorname{var}(\widehat{I C})=\frac{1}{N}\left[\sum_{t=1}^{T} f_{t} a_{t}^{2}-(1+I C)^{2}\right]+\frac{1}{N \mu^{2}} \sum_{t=1}^{T} f_{t} \sigma_{t}^{2}\left(2 R_{t}-1-I C\right)^{2}
$$

Donde $\mathrm{N}$ es el número total de observaciones $\mathrm{y}$

$$
a_{t}^{2}=\left(\frac{\mu_{t}}{\mu}\left[2 R_{t}-1-I C\right]+2-\frac{1}{\mu} \sum_{l=1}^{t-1} \mu_{l} f_{l}-\frac{1}{\mu} \sum_{l=1}^{t} \mu_{l} f_{l}\right)^{2}
$$

A partir de (2) los intervalos de confianza se estiman utilizando la formula usual:

$$
I C_{-}^{+}[H \sqrt{\operatorname{var}(\widehat{I C)}}]
$$

Donde la constante $\mathrm{H}$ es el 95 percentil en una distribución $t_{\mathrm{N}-1-1}$. 
Wagstaff (2005) demostró que los resultados obtenidos a partir de la ecuación (1) dependen del valor promedio de la variable salud $(\mu)$. Por lo tanto una comparación entre grupos con promedios diferentes llevaría a conclusiones equivocadas. En vista de eso, y con el fin de comparar las desigualdades entre los grupos de edad, se utiliza la modificación del IC propuesta por Erreygers (Erreygers, 2009) la cual no depende del promedio: el Índice de Concentración de Erreygers (ICE). El ICE se define como:

$$
I C E=4 \mu I C /(a-b)
$$

Donde a y $\mathrm{b}$ son el valor máximo y mínimo de la variable salud (O'Donnell et al., 2012). Tomando en cuenta que el $I C E$ es equivalente al $I C$ aplicado a la variable salud transformada linealmente (O'Donnell, et al., 2012), los valores obtenidos a partir de la ecuación (3) también se normalizan utilizando la transformación presentada en la ecuación (4).

\subsection{Descomposición del Î́ndice de Concentración}

Una vez estimado el $I C E$ y sus intervalos de confianza por edad, resta analizar los factores que explican el nivel de desigualdad en salud. Con este fin se han seleccionado un conjunto de factores que se reconocen como determinantes de la salud y que varían a través de los diferentes niveles de ingreso. La hipótesis que se plantea es que estos factores contribuyen a explicar las desigualdades en salud.

- Educación: La práctica de hábitos de vida saludables está asociada tanto a altos niveles de ingresos como de educación. Por esta razón que en múltiples estudios de desigualdad, se ha analizado las diferencias por nivel educacional en lugar de nivel de ingreso (Bago et al., 2008; Huisman et al., 2003). En el caso de Costa Rica, los adultos mayores tienen un promedio de educación muy inferior al del resto del país. Esto se debe a que las posibilidades de asistir a la educación primaria existentes antes de los años sesenta eran menores a las actuales (Robles y Rodríguez, 2008). Esto significa que tener el grado de educación básica durante el periodo en el que los adultos mayores eran parte de la fuerza laboral, permitía tener acceso a oportunidades que con el mismo nivel de educación no son posibles de alcanzar actualmente. En relación a la educación la hipótesis que se plantea es que la educación influye aumentando las desigualdades en salud medidas a través del ingreso. Adicionalmente, se espera diferencias por edad en la contribución que los niveles adicionales de educación aportan a la desigualdad en salud.

- Obesidad: Uno de los factores que se reconoce como de alto riesgo para la salud es la obesidad. Esta se encuentra positivamente relacionada con enfermedades crónicas. En Costa Rica la obesidad es un problema de salud 
pública, más de un $77 \%$ de las mujeres entre 45 y 65 años y un $62 \%$ de los hombres entre 20 y 64 años padecen de obesidad o sobrepeso (Ministerio de Salud de Costa Rica, 2012). En el caso de los adultos mayores la situación no es menos preocupante, el porcentaje de personas con obesidad o sobrepeso es de $61 \%$ en mujeres y $57 \%$ en los hombres (Ministerio de Salud de Costa Rica, 2012). En relación al nivel ingreso, a pesar de no existir un consenso general, una parte importante de la comunidad científica continua apoyando la idea de que existe una relación entre este y el sobrepeso, donde las personas con menores ingresos son más propensas a la obesidad (Drewnowski y Specter, 2004; Lakdawalla y Philipson, 2009). Se espera que la obesidad contribuya significativamente aumentando las desigualdades en salud medida a través del ingreso.

- Ejercicio Físico y Actividad en el Trabajo: Se ha comprobado que el ejercicio físico tiene la habilidad de reducir directamente los factores de riesgo de enfermedades crónicas y potenciar cambios positivos con respecto a otros factores de riesgo para estas enfermedades (Mora et al., 2004; Ramírez y Aragón, 1997). Las personas con mayores ventajas socioeconómicas tienden a dedicar mayor cantidad de tiempo a hacer ejercicio físico, relación que se explica en parte por las diferencias entre los grupos socioeconómicos en capital social y recursos disponibles (Estabrooks et al., 2003; Giles-Corti y Donovan, 2002). Por otra parte, Dosemeci et al. (1993) encontraron que mayores niveles de actividad física en el trabajo tienen un efecto protector contra el padecimiento de diferentes tipos de cáncer.

- Consumo de Tabaco: Las enfermedades que se relacionan al fumador tienen elevados costos para el sistema de salud costarricense (IAFA, 2009). Asimismo, las diferencias socioeconómicas en el consumo de tabaco están bien establecidas. Factores como la educación y el ingreso han sido encontrados inversamente relacionados al consumo de tabaco (Laaksonen et al., 2005; Stronks et al., 1997). A pesar de no existir consenso respecto a las causas de esta relación, la literatura sugiere como posible explicación la tendencia de las personas a fuman en respuesta al estrés, el cual puede ser inducido por situaciones socioeconómicas desfavorables.

- Relaciones Sociales, Satisfacción con la Atención que Recibe Cuando se Enferma: El interés en analizar el nexo entre las relaciones sociales y la salud de las personas mayores no es nuevo. Existe un gran número de investigaciones que demuestran esta relación tanto empíricamente como teóricamente a nivel de la salud cognitiva y física (Pinquart y Sörensen, 2000). La Primera Encuesta Nacional en Salud para Costa Rica (ENSA, 2006), fuente de los datos de este estudio, incluye un módulo denominado apoyo afectivo y personal. En este módulo se le pregunta al encuestado 
que tan satisfecho esta con sus relaciones sociales medidas estas a través: cariño y afecto que recibe, si es escuchado por alguien cuando tiene problemas, si recibe ayuda cuando está enfermo, entre otros (ver detalles en sección 2.3, Tabla 1). Entre las preguntas de este módulo existe una correlación positiva y significativa. Dado lo anterior y el hecho que parte de la importancia para la salud de las relaciones sociales descansa en el apoyo que brindan cuando hay deterioro de la salud, tanto para ayudar en la recuperación como para mantener el ánimo del paciente, se utiliza la pregunta "Recibe ayuda cuando está enfermo" para aproximar la satisfacción con las relaciones sociales. Asimismo, en el caso de los adultos mayores la decisión de utilizar la atención que recibe cuando se enferma, se ve respaldada por lo encontrado por Poga et al. (2007), quienes observaron que las redes sociales costarricenses muestran una mayor fortaleza en especial cuando empeora la salud de los adultos mayores. Por último, los datos de la ENSA suguieren que el número de adultos mayores de 60 años que consideran que no reciben ninguna o mucho menos de lo que desean disminuye con forme aumenta el ingreso.

La metodología propuesta por Rao (1969) y aplicada a la descomposición del IC por van Doorslaer et al. (2004) y por Wagstaff et al. (2003) permite descomponer la desigualdad como la suma ponderada de los factores que la determinan. En primer lugar se supone la existencia de un modelo lineal el cual explica la variable salud $\left(y_{i}\right)$ a partir del nivel de ingreso $\left(x_{I i}\right)$ y un conjunto de k-1 variables independientes (en este caso $\mathrm{k}-1=8$ donde estas ocho variables son: educación, obesidad, ejercicio físico, actividad en el trabajo, consumo de tabaco y satisfacción con la atención que recibe cuando se enferma, sexo y zona rural/urbano) tal que:

$$
y_{i}=\alpha_{0}+\alpha_{1} x_{I i}+\sum_{j=2}^{k} x_{j} x_{j i}+\varepsilon_{i}
$$

Donde $\alpha_{j}$ son los coeficientes a estimar y $\varepsilon_{\mathrm{i}}$ es un término de error. Se supone que cada individuo de la muestra independientemente del ingreso enfrenta los mismos coeficientes.

A partir de ecuación (7) el ICE para la variable salud se descompone de la siguiente manera:

$$
I C E=\left(\frac{\alpha_{1} \bar{x}_{I}}{\mu}\right) I C E_{I}+\sum_{j=2}^{k}\left(\frac{\alpha_{j} \bar{x}_{j}}{\mu}\right) I C E_{j}+G
$$

Donde $\mu$ continua representando el valor promedio de la variable salud, $\overline{x_{I}}$ $\mathrm{y} \overline{x_{j}}$ corresponden al promedio del ingreso $\left(x_{I}\right)$ y las variables $x_{j}$ respectivamente. $I C E_{j}$ es el índice de concentración para la variable independiente $j$ $(j=1, \ldots, \mathrm{k}-1)$ y revela la distribución socioeconómica de la variable independiente $j$. El mismo se estima a partir de la ecuación (1) la transformación en (2) sustituyendo $y_{i}$ por la variable $x_{j}$, lo mismo aplica con $I C E_{I}$. Por último, $G$ es 
un índice de concentración de los residuos, al que vamos a llamar término residual, el cual incluye tanto el error en la predicción como el error generado por utilizar una aproximación lineal para obtener los efectos marginales.

Pese a que $y_{i}$ es por lo general dicótoma la ecuación (5) se estima a partir de un modelo lineal utilizando mínimos cuadrados ordinarios (MCO). Lo anterior se debe a que la metodología utilizada para descomponer el índice supone que la desigualdad en salud puede descomponerse como la suma ponderada de los aportes de cada uno de los factores que la explican (ecuación (7)), donde para el ponderador se asume que existe una relación lineal entre la variable independiente y la medida de salud. Sin embargo, el ponderador, y por consiguiente la descomposición, dependen del parámetro $\alpha \mathrm{y}$ no de las probabilidades calculadas a partir de la ecuación (5), por tanto encontrar probabilidades fuera del rango $[0,1]$ no es importante para la descomposición del ICE (Balsa et al., 2009).

En la ecuación (6) la medida de concentración se descompone en tres fuentes de desigualdad. En primer lugar, la contribución del ingreso, la cual se define como el producto de la elasticidad $\left(\alpha_{1}\right)$ (evaluado en el promedio de la muestra $\left(\bar{x}_{I} / \mu\right)$ ) y el índice de concentración del ingreso. En segundo lugar, la contribución de las k-1 variables explicativas. Estas variables deben cumplir con dos requisitos, estar relacionadas al estado de salud $\left(\alpha_{j} \neq 0\right)$ y estar distribuidas de manera desigual según el nivel de ingreso $\left(I C E_{j} \neq 0\right)$, de lo contrario la contribución será igual a cero. En tercer lugar, un componente residual, el cual refleja la desigualdad en salud que no puede ser explicada por la variación en las variables independientes a través de los grupos de ingreso (Wagstaff et al., 2003).

Las estimaciones descritas en las secciones 2.1 y 2.2 se efectúan utilizando el programa $\mathrm{R}$ versión 3.0.0.

\subsection{Datos}

Se utilizan los datos de la Primera Encuesta Nacional en Salud para Costa Rica (ENSA, 2006) aplicada a una muestra aleatoria de población no institucionalizada de 2004 hogares. La sub-muestra seleccionada es de 4741 individuos y corresponde a las personas con edades por encima de 18 años. No existe un consenso en la literatura sobre a partir de cual edad una persona debe ser considerada adulto mayor, lo común es utilizar 60 o 65 años. En este caso se utiliza 60 años, punto de corte tomado en cuenta en la ENSA para efectuar el módulo adulto mayor y el cual es además el corte utilizado por las Naciones Unidas. Sin embargo, tratar a este grupo de edad como homogéneo sería incorrecto. Entre más edad es más probable encontrar mayores índices de morbilidad, una menor educación y mayores tasas de utilización de los servicios de salud. Por esta ra- 
zón, se divide la población de adultos mayores en tres segmentos de edad: de 60 a 69 , de 70 a 79 y de 80 a más.

En relación al nivel de ingreso, como lo que interesa es el nivel socioeconómico del encuestado y no su salario, se utiliza el ingreso familiar total reportado. Este incluye todas las posibles fuentes de ingreso (trabajo, ingreso privado, pensión, etc.). Como se trata de ingreso reportado, este corresponde más a una aproximación del nivel de ingreso y no al ingreso verdadero. Además, con el fin de considerar el tamaño de la familia, las estimaciones se hacen utilizando el ingreso per-cápita familiar ajustado a través de la escala de equivalencia de la $\mathrm{OECD}^{2}$ (Hagenaars et al., 1994). Este ajuste refleja el hecho de que el gasto promedio por miembro del hogar para cubrir las necesidades decrece cuando aumenta el número de miembros en el hogar. El mismo valor de ingreso se repite entre algunos individuos. Sin embargo, el análisis se basa en un número suficientemente elevado de grupos (entre 120 y 554 dependiendo del grupo de edad) tal que la perdida de información por utilizar datos agrupados es despreciable (Clarke y Van Ourti, 2009).

Tres medidas de salud se seleccionaron con el fin de obtener una completa visión de la desigualdad en salud existente en Costa Rica. La Tabla 1 muestra la distribución por edad de las tres medidas de salud así como de las demás variables incluidas en el análisis. Debido a que el ICE no puede ser calculado a partir de una variable categórica, la variable percepción de la salud es transformada en una variable cardinal con distribución log-normal estándar (Wagstaff y Van Doorslaer, 1994). Esto resulta en una medida de salud continua donde el valor más alto corresponde al peor estado de salud reportado y el más bajo al mejor estado de salud. Los ICE asociados a estas tres medidas de salud reflejarán la acumulación de mala salud, esto significa que valores negativos del ICE representan desigualdades en salud donde los pobres reportan peor salud que los ricos.

Tabla 1

Variables Socioeconómicas, Demográficas y de Salud (Porcentajes por grupo de edad)

\begin{tabular}{ccrrrrrrrr}
\hline \multirow{2}{*}{ Variable } & \multirow{2}{*}{ Respuestas } & \multirow{2}{*}{ Total } & \multicolumn{8}{c}{ Grupos de Edad } \\
\cline { 4 - 10 } & & $\mathbf{1 8}$ a $\mathbf{3 0}$ & $\mathbf{3 0}$ a $\mathbf{3 9}$ & $\mathbf{4 0}$ a $\mathbf{4 9}$ & $\mathbf{5 0}$ a $\mathbf{5 9}$ & $\mathbf{6 0}$ a $\mathbf{6 9}$ & $\mathbf{7 0}$ a 79 & $\Rightarrow \mathbf{8 0}$ \\
\hline $\begin{array}{c}\text { Padece Alguna } \\
\text { Enfermedad Crónica }\end{array}$ & $\mathrm{Si}$ & 32,9 & 8,3 & 21,8 & 31,4 & 43,6 & 58,9 & 64,9 & 68,2 \\
\hline
\end{tabular}

\footnotetext{
${ }^{2}$ Ingreso per cápita familiar ajustado por la escala de equivalencia de la OECD Ingreso familiar total reportado 
Tabla 1 (continuación)

Variables Socioeconómicas, Demográficas y de Salud (Porcentajes por grupo de edad)

\begin{tabular}{|c|c|c|c|c|c|c|c|c|c|}
\hline \multirow{2}{*}{ Variable } & \multirow{2}{*}{ Respuestas } & \multirow{2}{*}{ Total } & \multicolumn{7}{|c|}{ Grupos de Edad } \\
\hline & & & 18 a 30 & 30 a 39 & 40 a 49 & 50 a 59 & 60 a 69 & 70 a 79 & $\Rightarrow>80$ \\
\hline \multirow{5}{*}{$\begin{array}{l}\text { Percepción de la } \\
\text { Salud }^{\mathrm{b}}\end{array}$} & Muy malo & 1,0 & 1,0 & 0,8 & 0,7 & 0,4 & 1,4 & 1,8 & 2,0 \\
\hline & Malo & 3,6 & 1,2 & 2,2 & 2,4 & 6,1 & 6,2 & 5,4 & 10,1 \\
\hline & Regular & 22,7 & 14,3 & 20,3 & 21,8 & 22,1 & 28,9 & 38,8 & 45,5 \\
\hline & Bueno & 56,5 & 65,8 & 56,9 & 57,5 & 55,1 & 52,7 & 40,6 & 36,9 \\
\hline & Muy bueno & 15,9 & 17,7 & 19,4 & 17,2 & 16,0 & 10,6 & 13,4 & 5,6 \\
\hline \multirow[t]{3}{*}{$\begin{array}{l}\text { Limitación de } \\
\text { Actividad }^{c}\end{array}$} & $\mathrm{Si}$ & 46,6 & 28,7 & 36,5 & 45,5 & 54,0 & 66,7 & 71,3 & 75,8 \\
\hline & $\begin{array}{l}\text { De pie la mayor } \\
\text { parte del tiempo } \\
\text { pero sin efectuar } \\
\text { ningún esfuerzo. }\end{array}$ & 16,8 & 18,8 & 21,8 & 19,7 & 19,1 & 11,3 & 5,2 & 2,5 \\
\hline & $\begin{array}{l}\text { No realiza ninguna } \\
\text { actividad física. }\end{array}$ & 24,8 & 24,8 & 20,6 & 21,8 & 20,6 & 25,7 & 32,0 & 50,0 \\
\hline \multirow[t]{5}{*}{$\begin{array}{l}\text { Actividad Física en } \\
\text { el Trabajo }^{d}\end{array}$} & $\begin{array}{l}\text { De pie la mayor } \\
\text { parte del tiempo } \\
\text { pero sin efectuar } \\
\text { ningún esfuerzo. }\end{array}$ & 46,4 & 48,1 & 41,9 & 51,4 & 49,7 & 42,0 & 46,5 & 32,3 \\
\hline & $\begin{array}{l}\text { Caminando y } \\
\text { llevando peso. }\end{array}$ & 23,4 & 21,7 & 29,7 & 21,6 & 24,3 & 27,5 & 19,6 & 11,6 \\
\hline & $\begin{array}{l}\text { Realiza tareas que } \\
\text { requieren gran } \\
\text { esfuerzo físico. }\end{array}$ & 5,4 & 5,3 & 7,8 & 5,2 & 5,3 & 4,8 & 1,8 & 6,1 \\
\hline & $\begin{array}{l}\text { Ninguno o primaria } \\
\text { incompleta }\end{array}$ & 40,0 & 15,9 & 36,1 & 33,4 & 45,5 & 61,3 & 78,0 & 87,4 \\
\hline & $\begin{array}{l}\text { Primaria completa } \\
\text { o secundaria } \\
\text { incompleta }\end{array}$ & 37,4 & 54,5 & 36,3 & 43,6 & 30,7 & 24,6 & 13,4 & 9,6 \\
\hline \multirow{2}{*}{ Educación } & $\begin{array}{l}\text { Secundaria } \\
\text { completa o menos } \\
\text { de } 4 \text { años de } \\
\text { universidad o } \\
\text { parauniversidad }^{e}\end{array}$ & 4,7 & 10,5 & 4,4 & 2,5 & 2,9 & 1,1 & 0,5 & 0,5 \\
\hline & $\begin{array}{l}\text { No realiza ninguna } \\
\text { actividad o lo hace } \\
\text { menos de una vez } \\
\text { al mes }\end{array}$ & 59,8 & 54,6 & 61,1 & 53,7 & 62,7 & 64,9 & 64,9 & 79,8 \\
\hline Ejercicio Físico & $\begin{array}{l}\text { Actividad física o } \\
\text { deportiva al menos } \\
\text { una vez a la }\end{array}$ & 40,2 & 45,3 & 38,9 & 46,3 & 37,3 & 35,1 & 35,1 & 20,2 \\
\hline Fuma & $\begin{array}{l}\text { Fuma diaria y no } \\
\text { diariamente }\end{array}$ & 15,9 & 13,9 & 18,1 & 18,8 & 13,9 & 17,2 & 15,2 & 13,1 \\
\hline $\begin{array}{l}\text { Ingreso del Hogar } \\
\text { (Promedio) }\end{array}$ & $\begin{array}{l}\text { Ingreso percápita } \\
\text { familiar ajustado } \\
\text { por la escala de } \\
\text { equivalencia de la } \\
\text { OECD }\end{array}$ & 402,0 & 138,9 & 152,8 & 134,0 & 150,3 & 133,0 & 110,3 & 103,3 \\
\hline \multirow{3}{*}{$\begin{array}{c}\text { Obesidad: "En } \\
\text { relación a su } \\
\text { estatura, diría que } \\
\text { pesa" }\end{array}$} & $\begin{array}{l}\text { Menos que la de } \\
\text { su misma edad }\end{array}$ & 5,0 & 4,2 & 6,0 & 3,6 & 4,4 & 3,9 & 9,3 & 9,1 \\
\hline & $\begin{array}{l}\text { Es Igual que otros } \\
\text { de su edad }\end{array}$ & 56,0 & 61,2 & 51,5 & 54,2 & 50,7 & 55,0 & 56,8 & 65,7 \\
\hline & $\begin{array}{l}\text { Es más que de su } \\
\text { misma edad }\end{array}$ & 28,3 & 22,9 & 31,9 & 31,9 & 35,2 & 31,7 & 21,4 & 15,7 \\
\hline
\end{tabular}


Tabla 1 (continuación

Variables Socioeconómicas, Demográficas y de Salud (Porcentajes por grupo de edad)

\begin{tabular}{|c|c|c|c|c|c|c|c|c|c|}
\hline \multirow{2}{*}{ Variable } & \multirow{2}{*}{ Respuestas } & \multirow{2}{*}{ Total } & \multicolumn{7}{|c|}{ Grupos de Edad } \\
\hline & & & 18 a 30 & 30 a 39 & 40 a 49 & 50 a 59 & 60 a 69 & 70 a 79 & $\Rightarrow>80$ \\
\hline \multirow{3}{*}{$\begin{array}{l}\text { Atención que recibe } \\
\text { cuando se enferma }^{f}\end{array}$} & $\begin{array}{l}\text { Ninguno o mucho } \\
\text { menos de lo que } \\
\text { desea. }\end{array}$ & 5,8 & 4,0 & 6,3 & 6,4 & 6,6 & 6,2 & 6,5 & 8,1 \\
\hline & $\begin{array}{l}\text { Bastante pero } \\
\text { menos de lo que } \\
\text { desea. }\end{array}$ & 4,0 & 4,4 & 4,1 & 4,1 & 4,1 & 4,3 & 2,1 & 4,0 \\
\hline & $\begin{array}{l}\text { Tanto como desea } \\
\text { y más de lo que } \\
\text { desea. }\end{array}$ & 87,8 & 88,3 & 88,3 & 86,4 & 86,7 & 88,3 & 89,7 & 87,4 \\
\hline Sexo & Hombre & 46,7 & 47,3 & 52,7 & 43,8 & 44,3 & 47,5 & 44,4 & 43,4 \\
\hline Zona & Rural & 33,9 & 34,9 & 36,6 & 35,8 & 33,3 & 29,8 & 29,2 & 32,3 \\
\hline
\end{tabular}

a Lista de enfermedades consideradas crónicas: alergias, epilepsia, anemia, hemorroides, artrosis, reumatismo (incluso gota), dolores de espalda, hipertensión arterial, asma, bronquitis crónica o enfisema, jaquecas, migrañas o dolores de cabeza, cáncer, tumores, colesterol elevado, osteoporosis, diabetes, problemas psíquicos (depresión, nervios, alcoholismo, drogas), enfermedades de la sangre y ganglios linfáticos (anemia, leucemia), enfermedades del corazón y aparato circulatorio, enfermedades neurológicas (Párkinson, temblores).

b Pregunta: Ahora nos interesa saber de su estado de estado de salud, por lo que le vamos a hacer algunas preguntas, sería tan amable en decirme si en los últimos 12 meses, diría que su estado de salud ha sido muy bueno, bueno, regular o malo o muy malo? .

c Pregunta: ¿Ha tenido en los últimos 12 meses alguna enfermedad, o algún problema de salud que le haya limitado su actividad diaria por más de 10 días seguidos?.

d Pregunta: ¿Cuál de estas posibilidades describe mejor su actividad principal en el centro de trabajo, centro de enseñanza, hogar (labores domésticas), etc.?.

e Parauniversitaria: instituciones de educación superior que ofrecen carreras de dos a tres años de duración a personas egresadas de la educación secundaria, en donde se obtiene el título de diplomado. Universitaria: instituciones donde se obtienen títulos de bachillerato, licenciatura, maestría, especialidad y doctorado.

f Pregunta: De la siguiente lista de situaciones que pueden pasar, por favor, indique el grado del apoyo que recibe en cada una de ellas: Recibe ayuda cuando está enfermo en la cama. Esta variable está positiva y significativamente correlacionada con 5 de las 7 medidas de satisfacción del módulo Apoyo Afectivo y Personal de la ENSA 2006, estas variables son: 1) recibe cariño y afecto 2)tiene posibilidad de hablar con alguien de sus problemas (personales, familiares, etc.), bien sea con amigos, en su trabajo, en su casa, etc. 3)hay personas que se preocupan de lo que le sucede 4)recibe consejos útiles cuando le pasa algún acontecimiento importante en su vida 5)tiene buenas amistades.

Fuente: Elaboración propia. Datos: ENSA 2006.

Debido a que la ENSA 2006 no incluye una medida objetiva para medir obesidad, esta se aproxima utilizando la pregunta "En relación a su estatura, diría que pesa: más/menos/igual que otros de su edad". Se recomienda incluir en la ENSA una medida más objetiva que carezca de los problemas de sesgo asociados al auto-reporte del peso.

\section{RESULTADOS}

Antes de iniciar el análisis de resultados es importante recordar que las variables de salud a utilizar corresponden a medidas de estados de salud negativos. En otras palabras, para las dos medidas de salud dicótomas, una persona que 
reporta un estado salud negativo, o sea que padece alguna enfermedad crónica o ha visto su actividad limitada, se representa con el valor 1. Asimismo, para la medida de salud continua, percepción de la salud, entre mayor es el valor reportado por el individuo peor es la percepción de la salud. Dado que el ICE es una medida de concentración de la variable de interés, en este caso estados negativos de salud, valores negativos del ICE indican que la mayor proporción de mala salud se acumula en los sectores más pobres de la población. La Figura 2 muestra los valores de los Índices de Concentración de Erreygers para las tres medidas de salud por grupo de edad. Existen evidencias de desigualdad en salud en perjuicio de los más pobres. En términos generales las desigualdades se encuentran entre $-0,04$ y $-0,15$. Si se comparan con los ICEs del ingreso, entre 0,27 y 0,32 (el signo positivo indica una concentración de la riqueza en los niveles más altos de ingreso), podemos afirmar que la distribución de la salud es más equitativa que la del ingreso. La Figura 2 muestra además pocos indicios de desigualdad entre los adultos jóvenes.

Figura 2

Índices de Concentración de Erreygers por Grupos de Edad e Intervalos de Confianza

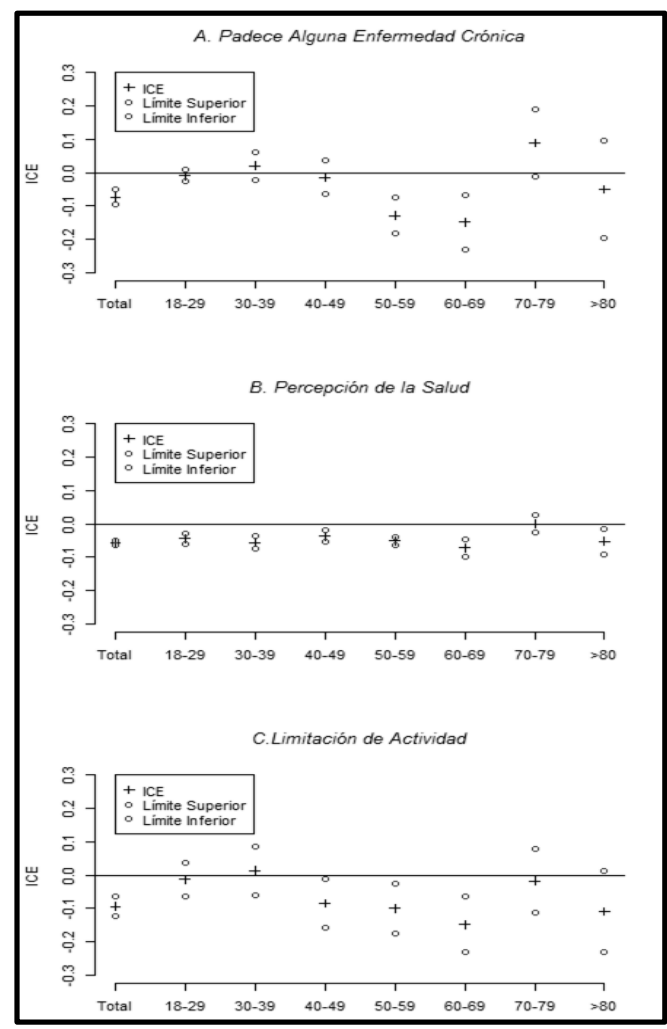

Fuente: Elaboración propia. 
En la medida de salud padece de alguna enfermedad crónica, dos grupos de edad muestran desigualdades significativas: 60-69 y 50-59 (Figura 2.A). Parte de este resultado se puede explicar por el crecimiento de la incidencia de enfermedades crónicas, como diabetes e hipertensión, que tiene su punto más alto en estos grupos de edad (Cheng y Ayala, 2004; Ministerio de Salud de Costa Rica et al., 2009). Los resultados reflejan el deterioro de los ingresos familiares producto de contraer un problema de salud crónico que afecta la capacidad para trabajar de los individuos entre 50 y 69 años de edad, lo cual tendría un impacto mayor en los hogares de bajos ingresos.

La percepción de la salud es la medida de salud con el mayor número de desigualdades significativas (Figura 2.B). Sin embargo, los ICE no superan el 0,07 en términos absolutos. Únicamente los adultos mayores entre 70 y 79 años presentan desigualdades no significativas. Para los mayores de 80 las desigualdades en la percepción de la salud son significativas lo que se contrapone a lo esperado. Sin embargo, esto deja de ser cierto cuando dividimos la población en urbana y rural, lo cual solo sucede en este grupo de edad (por razones de espacio los resultados del análisis por zona urbana y rural no se muestran).

Los resultados de la medida limitación de la actividad se presentan en la Figura 2.C. Conforme a lo esperado, los adultos mayores de 70 años presentan desigualdades no significativas.

En resumen, sin importar la medida de salud utilizada las desigualdades en salud más elevadas se observan en el grupo de 60 a 69 años. Este grupo se considera parte de los adultos mayores, sin embargo, los niveles de desigualdad en salud son similares a los observados en los adultos entre 50-59 años y no a los vistos en los mayores de 70 años. En Costa Rica para tener derecho a la pensión es necesario tener 65 años. Según el Censo del 2011, solo un 19\% de las personas entre 60 y 64 se encontraban pensionadas, cifra que crece a 34\% entre los 65 y 69 años y a $44 \%$ entre los 70 y 79 años. Dado que los adultos mayores pensionados tienen características diferentes, se estimó nuevamente el ICE dividiendo este grupo en dos: 60-64 y 65-69. Esto con el objetivo de identificar si el grupo 60-69 se compone en realidad de dos grupos diferentes en términos de inequidad, uno más similar a los adultos y otro más similar a los adultos mayores. Los costarricenses que presentan las mayores desigualdades en salud son los adultos mayores entre 65 y 69 años (Tabla 2). Las diferencias entre los nuevos pensionados de ingresos altos y los de ingresos bajos (por ejemplo: tipos de actividades diarias, prevención de enfermedades, consultas de salud, entre otros), podrían explicar estas desigualdades en salud. Dada la limitación de datos un análisis detallado de este resultado queda fuera de las posibilidades del estudio. 
Tabla 2

Inequidades en Salud para los Grupo de Edad 60-64 y 65-69

\begin{tabular}{llc}
\hline & \multicolumn{2}{c}{ Grupos de Edad $^{\mathrm{a}}$} \\
\cline { 2 - 3 } & $\mathbf{6 0 - 6 4}$ & $\mathbf{6 5 - 6 9}$ \\
\hline Padece Alguna Enfermedad Crónica & $-0,06$ & $-0,28^{*}$ \\
Percepción de la Salud & $-0,05^{\star}$ & $-0,10^{*}$ \\
Limitación de Actividad & $-0,13^{*}$ & $-0,18^{*}$ \\
Número de Observaciones & 334 & 230 \\
\hline
\end{tabular}

* Significativamente diferente de cero.

Fuente: Elaboración propia.

En relación a la descomposición de los $I C E$ s, en la Tabla 3 se observa que el aporte del ingreso es el principal responsable de las desigualdades en salud en perjuicio de los más pobres. Esto se debe a un alto valor positivo del $I C E_{I}$ y una relación lineal significativa y negativa entre la mala salud y el ingreso reflejada en el resultado del $\alpha_{1}$ (véase ecuación (6) en epígrafe 2.2) (por razones de espacio los ICEs y $\alpha$ de las variables explicativas no se muestran). Esto último indica que la probabilidad de estados de salud negativos decrece al aumentar el ingreso en todas las edades. Por su parte el término residual en casi todos los casos tiene una participación porcentual positiva. Lo que sugiere la existencia de un grupo de variables no incluidas que compensan el efecto negativo del ingreso. Por tanto, se recomienda ampliar el número de factores en el análisis con el fin de encontrar aquellos que contribuyen a disminuir las desigualdades pro-rico.

Tabla 3

Contribución Porcentual ${ }^{a}$ de los Factores Seleccionados a las Desigualdades en Salud Estadísticamente Significativas

\begin{tabular}{|c|c|c|c|c|c|c|c|}
\hline \multirow{2}{*}{ Variable $^{b}$} & \multicolumn{7}{|c|}{ Grupos de Edad $^{\mathrm{C}}$} \\
\hline & Total & $18-29$ & $30-39$ & $40-49$ & $50-59$ & $60-69$ & $>=80$ \\
\hline \multicolumn{8}{|c|}{ Limitación de la Actividad } \\
\hline Actividad Física en el Trabajo & 1,2 & - & - & $-0,1$ & $-0,4$ & 1,2 & - \\
\hline Educación & $-2,8$ & - & - & $-12,6$ & 27,1 & $-2,6$ & - \\
\hline Ejercicio Físico & 2,7 & - & - & $-1,5$ & 9,7 & 3,6 & - \\
\hline Fuma (Fuma diaria y no diariamente) ${ }^{d}$ & $-0,1$ & - & - & 0,0 & 0,5 & $-0,2$ & - \\
\hline $\begin{array}{l}\text { Ln Ingreso per-cápita familiar en miles de } \\
\text { colones }\end{array}$ & $-114,5$ & - & - & $-183,2$ & $-216,2$ & $-84,2$ & - \\
\hline $\begin{array}{l}\text { Peso en Comparación las Personas de la } \\
\text { Misma Edad }\end{array}$ & 0,2 & - & - & $-2,8$ & $-4,0$ & 7,4 & - \\
\hline $\begin{array}{l}\text { Satisfacción con la Atención que Recibe } \\
\text { Cuando se Enferma }\end{array}$ & 0,1 & - & - & 1,2 & $-0,2$ & 5,5 & - \\
\hline Sexo $(\text { Hombre })^{d}$ & $-2,6$ & - & - & $-4,2$ & $-3,0$ & 0,2 & - \\
\hline Zona (Rural) $^{d}$ & 3,6 & - & - & 8,2 & $-9,8$ & $-0,9$ & - \\
\hline Término Residual & 12,1 & - & - & 95,0 & 96,2 & $-30,1$ & - \\
\hline
\end{tabular}


Tabla 3 (continuación)

Contribución Porcentual ${ }^{a}$ de los Factores Seleccionados a las Desigualdades en Salud Estadísticamente Significativas

\begin{tabular}{|c|c|c|c|c|c|c|c|}
\hline \multirow{2}{*}{ Variable $^{b}$} & \multicolumn{7}{|c|}{ Grupos de Edad $^{c}$} \\
\hline & Total & $18-29$ & $30-39$ & $40-49$ & $50-59$ & $60-69$ & $>=80$ \\
\hline \multicolumn{8}{|c|}{ Padece Alguna Enfermedad Crónica } \\
\hline Actividad Física en el Trabajo & 5,5 & - & - & - & 11,1 & 3,6 & - \\
\hline Educación & $-18,1$ & - & - & - & 25,9 & $-3,5$ & - \\
\hline Ejercicio Físico & 4,3 & - & - & - & 10,0 & 6,7 & - \\
\hline Fuma (Fuma diaria y no diariamente) ${ }^{\mathrm{d}}$ & $-0,5$ & - & - & - & $-2,8$ & $-0,1$ & - \\
\hline $\begin{array}{l}\text { Ln Ingreso per-cápita familiar en miles de } \\
\text { colones }\end{array}$ & $-208,1$ & - & - & - & $-313,9$ & $-243,0$ & - \\
\hline $\begin{array}{l}\text { Peso en Comparación las Personas de la } \\
\text { Misma Edad }\end{array}$ & 0,9 & - & - & - & 5,9 & 9,9 & - \\
\hline $\begin{array}{l}\text { Satisfacción con la Atención que Recibe } \\
\text { Cuando se Enferma }\end{array}$ & $-18,6$ & - & - & - & $-35,1$ & 32,8 & - \\
\hline Sexo $(\text { Hombre })^{d}$ & $-4,2$ & - & - & - & $-5,3$ & 0,9 & - \\
\hline Zona (Rural) $^{\mathrm{d}}$ & 9,7 & - & - & - & $-7,4$ & $-1,6$ & - \\
\hline Término Residual & 129,2 & - & - & - & 211,5 & 94,3 & - \\
\hline \multicolumn{8}{|c|}{ Percepción de la Salud } \\
\hline Actividad Física en el Trabajo & 0,3 & $-1,8$ & $-4,6$ & 3,6 & $-6,7$ & $-0,8$ & 0,8 \\
\hline Educación & $-10,3$ & $-29,6$ & 87,9 & $-1,6$ & 34,1 & $-22,5$ & $-30,0$ \\
\hline Ejercicio Físico & $-2,3$ & 6,8 & $-1,4$ & $-8,0$ & $-5,0$ & 1,1 & 11,8 \\
\hline Fuma (Fuma diaria y no diariamente) ${ }^{d}$ & $-0,5$ & $-2,5$ & $-2,4$ & 0,1 & $-3,2$ & $-1,5$ & $-8,1$ \\
\hline $\begin{array}{l}\text { Ln Ingreso per-cápita familiar en miles de } \\
\text { colones }\end{array}$ & $-404,3$ & $-417,5$ & $-576,6$ & $-539,3$ & $-633,5$ & $-399,4$ & $-296,2$ \\
\hline $\begin{array}{l}\text { Peso en Comparación las Personas de la } \\
\text { Misma Edad }\end{array}$ & $-0,5$ & 0,8 & $-30,8$ & $-3,4$ & $-10,8$ & 3,5 & 44,5 \\
\hline $\begin{array}{l}\text { Satisfacción con la Atención que Recibe } \\
\text { Cuando se Enferma }\end{array}$ & 2,7 & $-15,7$ & 9,5 & $-19,9$ & 0,1 & 12,1 & $-7,4$ \\
\hline Sexo (Hombre) ${ }^{d}$ & $-3,4$ & $-10,3$ & $-3,0$ & $-1,4$ & $-3,3$ & $-2,9$ & $-26,5$ \\
\hline Zona (Rural) ${ }^{\mathrm{d}}$ & 2,7 & 2,9 & 2,5 & $-1,0$ & $-1,0$ & $-0,5$ & 32,7 \\
\hline Término Residual & 315,5 & 366,9 & 419,0 & 470,9 & 529,3 & 311,0 & 178,5 \\
\hline
\end{tabular}

${ }^{a}$ Contribución porcentual se refiere al aporte de la variable explicativa estimado en la ecuación (6) dividido por el

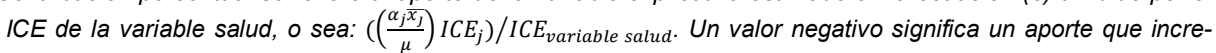
menta las desigualdades en salud en perjuicio de los más pobres. Entre las columnas no es posible hacer una comparación directa de los valores, ya que estos dependen del valor de ICE de la variable de salud, por tanto lo que se analiza es la importancia relativa que tiene cada factor dentro de la columna.

${ }^{b}$ Los valores que se muestran corresponden al aporte neto de cada variable, se calculan sumando los aportes de las subcategorías.

${ }^{c}$ Se muestran solamente los resultados de los grupos de edad que presentan desigualdades en salud (ICE) estadísticamente diferentes de cero.

Fuente: Elaboración propia.

Los resultados del factor educación merecen un análisis más profundo. En la Tabla 3 se muestra únicamente el aporte neto de la variable educación, el cual corresponde a la suma de los aportes de cada una de las categorías en las que se divide está variable. Los aportes individuales de cada categoría se muestran en la Tabla 4. Se distinguen diferencias entre las categorías en el signo y la magnitud del aporte. En la mayoría de los casos primaria completa o secundaria 
incompleta tiene un efecto positivo en la disminución de las desigualdades prorico, mientras que 4 años o más de universidad/para-universidad tiende a incrementar las desigualdades en salud. Esto se debe en primer lugar a la distribución por nivel de ingreso (medida a través de los $I C E$ s de cada categoría). El número de personas con 4 años o más de universidad/para-universidad es mayor entre los niveles altos de ingresos (ICEs positivos) y el número de personas con poca educación se concentra entre los niveles más pobres (ICEs negativos). Los $\alpha$, que miden la relación lineal entre la medida de salud y la categoría de educación, son todos negativos. Esto implica que, en comparación con las personas sin ninguna educación, cualquier nivel de educación disminuye las probabilidades de reportar problemas de salud. Al sumar las categorías, el aporte positivo de primaria completa o secundaria incompleta se ve superado por los valores negativos de las dos categorías superiores de educación. Además vale la pena señalar que los grupos de edad para los cuales la educación tiene el menor aporte a las desigualdades en salud son los adultos mayores, lo cual se relaciona a las menores oportunidades educativas que existían hace 50 años o más, cuando las diferencias educativas, independiente del ingreso, eran menores.

Tabla 4

Contribución Porcentual de las Categorías de la Variable Educación a las Desigualdades en Salud Estadísticamente Significativas

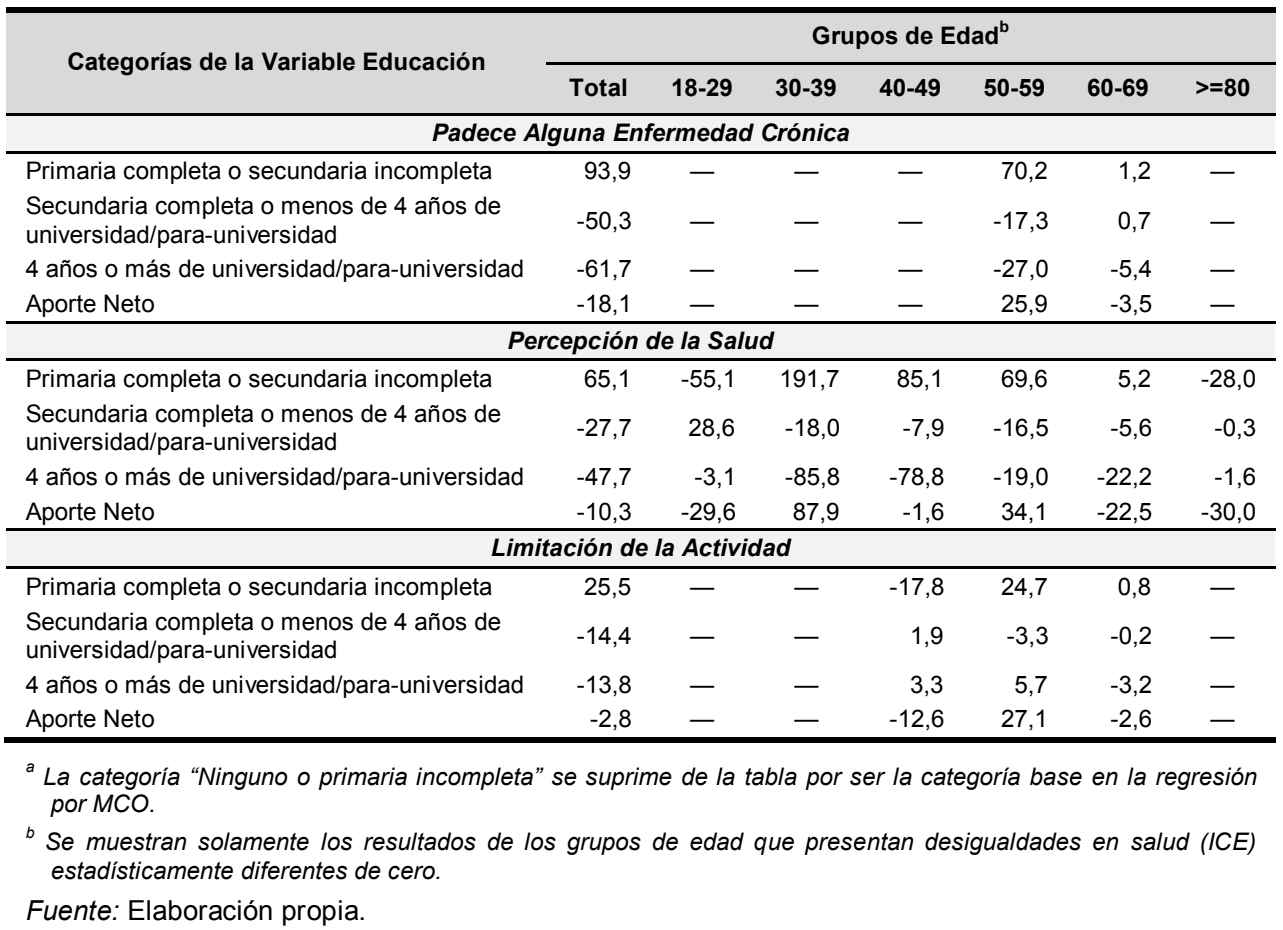


La obesidad resultó no tener un efecto significativo en el estado de salud. El resultado encontrado puede deberse a que la variable elegida para aproximar obesidad, peso en comparación a las personas de la misma edad, no permite analizar debidamente el efecto de la obesidad sobre la salud.

Finalmente, pese a que algunas de las variables explicativas utilizadas pudieran tener el riesgo de estar correlacionadas, los valores inferiores a 1,6 del Factor de Inflación de la Varianza (vif) indican que, sin importar grupo de edad o medida de salud, no existe un problema de correlación entre los factores explicativos que provoque un sesgo en los resultados.

\section{DISCUSIÓN}

Utilizando el Índice de Concentración ajustado por la recomendación de Erreygers se ha mostrado que existe en Costa Rica una desigualdad en salud que afecta negativamente a los más pobres.

Las desigualdades encontradas en las tres medidas de salud analizadas son menores o no significativas en los adultos mayores de 70 años. Este resultado se encuentra acorde con la literatura, la cual indica que los niveles de desigualdad se reducen entre los grupos de mayor edad. En el grupo de adultos mayores de 60 a 69 las desigualdades son las más elevadas de entre todos los grupos de edad. Dado que la tasa de nuevos pacientes diagnosticados con alguna enfermedad crónica es especialmente elevada en estas edades, el resultado evidencia la necesidad del sistema de salud costarricense de invertir en mejoras en el acceso y atención de los nuevos pacientes crónicos que pertenecen a los estratos bajos de ingreso. Asimismo, en el grupo donde se ubican los nuevos pensionados, entre 65 y 69, es donde la brecha en todas las medidas de salud es especialmente importante.

El ingreso es por mucho la principal fuente de desigualdades en salud. Lo que implica que disminuir las disparidades en el ingreso disminuirá las desigualdades en salud. Esto es de suma importancia ya que en los últimos años las desigualdades en ingreso y los niveles de pobreza han crecido considerablemente en Costa Rica. Lo cual sugiere que las desigualdades en salud también pueden haberse incrementado. Debido a que la ENSA se aplicó solamente para el año 2006 y no existe otra fuente de datos que permita el análisis de las tres medidas de salud aquí utilizadas, esta tesis no pudo ser analizada dentro de este documento. En vista de la evidente importancia del ingreso en la creación de desigualdades en salud, se recomienda una nueva aplicación de la ENSA y la estimación para el presente año de las medidas de desigualdad en salud.

El segundo factor clave es la educación, en especial la discrepancia entre el número de personas que asiste a la universidad por nivel de ingreso. Los resultados apuntan a que si bien la educación primaria universal y obligatoria, en la que Costa Rica ha basado gran parte de su crecimiento económico, ha tenido un 
impacto positivo en la disminución de las desigualdades en salud, no es suficiente para contrarrestar el efecto negativo que las desigualdades en el acceso a la educación secundaria y universitaria provocan. Por tanto, es necesario reforzar los resultados obtenidos en primaria mejorando el acceso de las personas de bajos ingresos a grados superiores de educación.

Es necesario mencionar tres limitaciones del estudio, primero el efecto de la correlación entre el ingreso y las demás variables explicativas en la precisión de las estimaciones de los coeficientes del modelo de MCO. En este sentido, cabe señalar que los resultados del vif son en todas las ecuaciones inferiores 1,6 , lo que indica que si existiera correlación esta es despreciable. La segunda limitación de este estudio es la falta de datos que permitan analizar las diferencias en las desigualdades por cohorte. Factores como por ejemplo, las diferencias en la estructura educativa entre los adultos y los adultos mayores, pueden estar explicando parte de los resultados. En cuanto a la tercera limitación, pese a que la ENSA es un corte transversal de la situación en 2006, es la única encuesta aplicada en Costa Rica donde es posible obtener información del ingreso, el estado de salud y los hábitos de vida. Por tanto una de las recomendaciones que se quiere enfatizar en este trabajo, es la necesidad de aplicar nuevamente la Encuesta Nacional de Salud y con esto poder mejorar nuestra comprensión de la relación entre el ingreso y la salud así como su evolución temporal.

En resumen, si bien el tratamiento de enfermedades es urgente y debe ser prioritario, no debe impedir tomar las medidas adecuadas tal que todos los niveles socioeconómicos se vean beneficiados con las mejoras en los indicadores de salud. Cuatro recomendaciones de política se sugieren con el fin de disminuir la brecha entre la salud de los ricos y pobres. En primer lugar, es necesario fortalecer el acceso y los servicios de salud para las personas entre 50 y 69 años con menores ingresos, donde la tasa de en enfermedades crónicas crece de manera importante. En segundo lugar, es preciso aumentar las probabilidades de acceso a la educación universitaria, factor mitigante de las desigualdades en salud que surjan del incremento en las desigualdades en ingreso. En tercer lugar, es importante considerar políticas de reducción de las desigualdades que se centren principalmente en el grupo de edad entre los 65 y 69 años quienes son los más vulnerables ante el incremento sustancial de las desigualdades en ingreso que sufre Costa Rica. Para finalizar es importante recalcar que cualquier intento de política que busque la igualdad pero que no tome en cuenta las variaciones en la desigualdad según grupo edad podría llevar a resultados no deseados. Este no es solo cierto para el caso costarricense, sino además para el resto del continente. En Latinoamérica el tema de las desigualdades en salud por edad ha sido pocas veces analizado. En una región con acelerado envejecimiento poblacional y donde los problemas de salud asociados consumen los recursos públicos cada vez con más voracidad, promover el análisis de las desigualdades 
por grupo de edad es fundamental para el fortaleciendo de los sistemas de salud y la disminución de las brechas entre ricos y pobres.

\section{REFERENCIAS BIBLIOGRÁFICAS}

ASAMBLEA LEGISLATIVA DE COSTA RICA (1973). Ley 5395 Artículos 1-3. En: Ley General de la Salud. San José, Costa Rica: República de Costa Rica.

BAGO, T.; O'DONNELL, O. y VAN DOORSLAER, E. (2008). "Differential Health Reporting by Education Level and Its Impact on the Measurement of Health Inequalities among Older Europeans". International Journal of Epidemiology, 37(6), pp. 13751383.

BALSA, A.; FERRES, D.; ROSSI, M. y TRIUNFO, P. (2009). "Inequidades Socioeconómicas en el Uso de los Servicios Sanitarios del Adulto Mayor Montevideano". Estudios Económicos, 24(1), pp. 35-88.

CAJA COSTARRICENSE DEL SEGURO SOCIAL (CCSS) (2013). Blog de Noticias: CCSS Tiene en Funcionamiento 1014 Ebáis en todo el Territorio Nacional. http://www.ccss.sa.cr/noticias/index/22-/980-ccss-tiene-en-funcionamiento1-014-ebais-en-todo-el-territorio-nacional. [Último acceso: Diciembre 2013].

CARDONA, D., DÉBORA, L. y BERTONE, C. (2013). "Inequidades en Salud entre Países de Latinoamérica y el Caribe”. Gaceta Sanitaria, 27(4), pp. 292-297.

CENTRO CENTROAMÉRICA DE POBLACIÓN (CCP) y INSTITUTO NACIONAL DE ESTADÍSTICA Y CENSOS (INEC) (2008). Estimaciones y Proyecciones de Población por Sexo y Edad: 1950 -2100. San José, Costa Rica.

CHENG, Y. y AYALA, N. (2004). Prevalencia de la Diabetes Mellitus en Costa Rica. San José, Costa Rica: Caja Costarricense de Seguro Social, Gerencia División Administrativa Dirección de Compra de Servicios de Salud.

CLARKE, P. y VAN QURTI, T. (2009). "Correcting the Bias in the Concentration Index When Income is Grouped". Journal of Health Economics, 29(1), pp. 151-157.

DEATON, A. y PAXSON, C. (1998). "Aging and Inequality in Income and Health". The American Economic Review, 88(2), pp. 248-253.

DOSEMECI, M.; HAYES, R.B.; VETTER, R.; HOOVER, R.N.; TUCKER, M.; ENGIN, K.; UNSAL, M. y BLAIR, A. (1993). "Occupational Physical Activity, Socioeconomic Status, and Risks of 15 Cancer Sites in Turkey". Cancer Causes \& Control, 4(4), p. 313321.

DREWNOWSKI, A. y SPECTER, S. (2004). "Poverty and Obesity: The Role of Energy Density and Energy Costs". The American Journal of Clinical Nutrition, 79(1), pp. 616.

ERREYGERS, G., (2009). "Correcting the Concentration Index". Journal of Health Economics, 28(2), pp. 504-515.

ESTABROOKS, P.; LEE, R. y GYURCSIK, N. (2003). "Resources for Physical Activity Participation: Does Availability and Accessibility Differ by Neighborhood Socioeconomic Status?". Annals of Behavioral Medicine, 265(2), pp. 100-104. 
ESTADO DE LA NACIÓN (2012). "Equidad e Integración Social". En: Decimooctavo Informe Estado de la Nación en Desarrollo Humano Sostenible (pp. 39). San José, Costa Rica: Programa Estado de la Nación en Desarrollo Humano Sostenible.

ESTADO DE LA NACIÓN (2013): Demografía y Desarrollo Humano. San José, Costa Rica: Estado de la Nación. http://www.estadonacion.or.cr/estadisticas/costarica/compendio-estadistico/estadisticas-sociales ['Ultimo Acceso: Marzo 2013]

FERNÁNDEZ, X. y ROBLES, A. (2008a). "Uso y Acceso a los Servicios de Salud". En: I Informe Estado de Situación de la Persona Adulta Mayor en Costa Rica (pp. 125148). San José, Costa Rica: Universidad de Costa Rica.

FERNÁNDEZ, X. y ROBLES, A. (2008b). "Seguridad Económica". En: I Informe Estado de Situación de la Persona Adulta Mayor en Costa Rica (pp. 125-148). San José, Costa Rica: Universidad de Costa Rica.

GILES-CORTI, B. y DONOVAN, J. (2002). "Socioeconomic Status Differences in Recreational Physical Activity Levels and Real and Perceived Access to a Supportive Physical Environment". Preventive Medicine, 35(6), pp. 601-611.

GRUNDY, E. y SLOGGETT , A. (2003). "Health Inequalities in the Older Population: The Role of Person al Capital, Social Resources and Socio-economic Circumstances". Social Science \& Medicine, 56(5), pp. 935-947.

HAGENAARS, A.; DE VOS, K. y ZAIDI , M. (1994). Poverty Statistics in the Late 1980s: Research Based on Micro-data. Luxembourg: Office for Official Publications of the European Communities.

HERNÁNDEZ-QUEVEDO, C. y MASSERIA, C. (2013). "Midiendo las Desigualdades en Salud Relacionadas con la Renta entre Países". Estudios de Economía Aplicada, 31(2), pp. 455-476.

HERRERO, F. y DURÁN, F. (2001). El Sector Privado en el Sistema de Salud de Costa Rica. Naciones Unidas: Comisión Económica para América Latina y el Caribe (CEPAL).

HUISMAN, M.; KUNST, A. y MACKENBACH, J. (2003). "Mackenbach Socioeconomic Inequalities in Morbidity among Elderly a European Overview". Social Science \& Medicine, 57(5), pp. 861-873.

INSITUTO NACIONAL DE ESTADISTICA Y CENSOS (INEC) (2011). Cuadro 9: Costa Rica: Población total por condición de aseguramiento y tipo de seguro social, según provincia, cantón y sexo. http://www.inec.go.cr/Web/Home/GeneradorPagina.aspx. ['Ultimo Acceso: Enero 2013]

INSTITUTO SOBRE ALCOHOLISMO Y FARMACODEPENDENCIA (IAFA) (2009). Consumo de Drogas en Costa Rica: Resultados de la Encuesta Nacional 2006. San José, Costa Rica: IAFA.

JENKINS, S. (1988). "Calculating Income Distribution Indexes from Microdata". National Tax Journal, 41(1), p. 139-142.

KAKWANI, N. (1980). Income Inequality and Poverty: Methods of Estimation and Policy Applications. New York: Oxford University Press.

KAKWANI, N.; WAGSTAFF, A. y VAN DOORSLAER, E. (1997). "Socioeconomic Inequalities in Health: Measurement, Computation and Statistical Inference". Journal of Econometrics, 77(1), pp. 87-103.

KNAUL, F.M.; WONG, R.; ARREOLA-ORNELAS, H. y MÉNDEZ, O. (2011). "Household Catastrophic Health Expenditures: A Comparative Analysis of Twelve Latin American and Caribbean Countries". Salud Pública México, 53(2), pp. 85-95. 
LAAKSONEN, M.; RAHKONEN, O.; KARVONEN, S. y LAHELMA, E. (2005). "Socioeconomic Status and Smoking Analysing Inequalities with Multiple Indicators". European Journal of Public Health, 15(3), p. 262-269.

LAKDAWALLA, D. y PHILIPSON, T. (2009). "The Growth of Obesity and Technological Change". Economics and Human Biology, 7(3), p. 283-293.

MACKENBACH, J.P.; STIRBU, I.; ROSKAM, A.; SCHAAP, M.M.; MENVIELLE, G.; LEINSALU, M. y ANTON E. KUNST A.E. (2008). "Socioeconomic Inequalities in Health in 22 European Countries". The New England Journal of Medicine, 358(23), pp. 2468-2481.

MINISTERIO DE SALUD DE COSTA RICA (2012). Encuesta Nacional de Nutrición de Costa Rica, 2008-2009. ANTROPOMETRIA. http://www.ministeriodesalud.go.cr/ index.php/informacion/doc_details/1028-en-cuesta-nacional-de-nutricion-2008-2009antropometria?tmpl=component ['Ultimo Acceso: Enero 2013]

MINISTERIO DE SALUD DE COSTA RICA, CAJA COSTARRICENSE DE SEGURO SOCIAL (CCSS), INSTITUTO COSTARRICENSE DE INVESTIGACIÓN Y ENSEÑANZA EN NUTRICIÓN Y SALUD y ORGANIZACIÓN PANAMERICANA DE LA SALUD (OPS), (2009). Encuesta Multinacional de Diabetes Mellitus, Hipertensión Arterial y Factores de Riesgo Asociados Área Metropolitana, San José, 2004. San José, Costa Rica.

MORA, M.; VILLALOBOS, D.; ARAYA VARGAS, G. y OZOLS, A. (2004). "Perspectiva Subjetiva de la Calidad de Vida del Adulto Mayor, Diferencias Ligadas al Género y a la Práctica de la Actividad Físico Recreativa". Movimiento Humano y Salud, 1(1), pp. 1-12.

MORERA, M. (2009). "Inequidad en Salud en Costa Rica: Una Realidad que no Debemos Ignorar". Revista Costarricense de Salud Pública, Volumen 18(2), pp. 64-65.

MURRAY, C.J.L.; KULKARNI, S.C.; MICHAUD, C.; TOMIJIMA, N.; BULZACCHELLI, M.T.; IANDIORIO, T.J. y EZZATI, M. (2006). "Eight Americas: Investigating Mortality Disparities across Races, Counties, and Race-Counties in the United States". PloS Medicine, 3(9), pp. 1513-1524.

O'DONNELL, O.; VAN DOORSLAER, E. y WAGSTAFF, A. (2012). "Chapter 17: Decomposition of Inequalities in Health and Health Care". En Andrew M. Jones (ed.): The Elgar Companion of Health Economics (pp. 179-191). Northampton, MA, USA: Edward Elgar Publishing.

O'DONNELL, O.; VAN DOORSLAER, E.; WAGSTAFF, A. y LINDELOW, M. (2008). Analyzing Health Equity Using Household Survey Data: A Guide to Techniques and Their Implementation. Washington, DC: The International Bank for Reconstruction and Development / The World Bank.

ORGANIZACIÓN MUNDIAL DE LA SALUD (OMS) (2000). "Logro y Desempeño del sistema de Salud en Todos los Estados Miembros". En: Informe Sobre la Salud en el Mundo 2000 - Mejorar el Desempeño de los Sistemas de Salud (pp. 176-179). Ginebra, Suiza.

PINQUART, M. y SÖRENSEN, S. (2000). "Influences of socioeconomic status, social network, and competence on subjective well-being in later life: A meta-analysis". Psychology and Aging, 15(2), pp. 187-224.

POGA, D.; ROSERO-BIXBY, L.; GLASER, K. y CASTRO, T. (2007). "Red Social y Salud del Adulto mayor en Perspectiva Comparada: Costa Rica, España e Inglaterra”. Población y Salud en Mesoamérica, 5(1), pp. 1-21. 
RAMíREZ, J. y ARAGÓN, L. (1997). "Efectos del Ejercicio Físico en la Funcionalidad de Mujeres Mayores". Revista Costarricense de Salud Pública, 6 (11), pp. 33-42.

RAO, V. (1969). "Two Decompositions of Concentration Ratio". Journal of the Royal Statistical Society, 132(3), pp. 418-425.

ROBLES, A. y RODRÍGUEZ, M. (2008). “¿Quiénes son y Dónde Están los Adultos Mayores?". En Fernández, X. y Robles, A. (ed.): I Informe estado de situación de la persona adulta mayor en Costa Rica (pp. 11-22). San José, Costa Rica: Universidad de Costa Rica (UCR).

ROSERO, L. (2004). "Evaluación del Impacto de la Reforma del Sector Salud en Costa Rica Mediante un Estudio Cuasi-experimental". Revista Panamericana de Salud Pública, 15(2), pp. 94-103.

SAÉNZ, M.; ACOSTA, M.; MUISER, J. y BERMÚDEZ, J. (2011)." Sistema de Salud de Costa Rica". Salud Pública de México, 53(2), pp. 156-167.

STRONKS, K.; VAN DE MHEEN, H.; LOOMAN, C. y MACKENBACH, J. (1997). "Cultural, Material, and Psychosocial Correlates of the Socio-economic Gradient in Smoking Behavior among Adults". Preventive Medicine, 26 (1), pp. 754-766.

VAN DOORSLAER, E.; KOOLMAN, X. y JONES, A. (2004). "Explaining Income-Related Inequalities in Doctor Utilization in Europe". Health Economics, 13(7), pp. 629-647.

VAN OURTI, T. (2003). "Socio-economic Inequality in III-health amongst the Elderly. Should one use Current or Permanent Income?". Journal of Health Economics, 22(2), pp. 219-241.

WAGSTAFF, A. (2005). "The Bounds of the Concentration Index When the Variable of Interest is Binary, With an Application to Immunization Inequality". Health Economics, 14(4), pp. 429-432.

WAGSTAFF, A.; PACT, P. y VAN DOORSLAER, E. (1991). "On The Measurement of Inequalities in Health". Social Science \& Medicine, 33(5), pp. 545-557.

WAGSTAFF, A. y VAN DOORSLAER, E. (1994). "Measuring Inequalities in Health in the Presence of Multiple-Category Morbidity Indicators". Health Economics, 3(4), pp. 281-9.

WAGSTAFF, A.; VAN DOORSLAER, E. y WATANABE, N. (2003). "On Decomposing the Causes of Health Sector Inequalities, with an Application to Malnutrition Inequalities in Vietnam". Journal of Econometrics, 112(1), pp. 219-227.

WAGSTAFF, A. y WATANABE, N. (2003). "What difference does the Choice of SES Make in Health Inequality Measurement?". Health Economics, 12(10), pp. 885-890. 